\title{
Políticas de habitabilidad en calle en Bogotá, Colombia, ¿hacia el desarrollo humano integral?
}

Homelessness policies in Bogotá, Colombia, towards integral human development?

Luisa Fernanda Zamudio Rochal Universidad Santo Tomás (Colombia)

Artículo de investigación

Fecha de recepción: Abril 2 de 2018

\section{Para citar este artículo}

Zamudio, L. F. (2018). Políticas de habitabilidad en calle en Bogotá, Colombia, ¿hacia el desarrollo humano integral? Revista Campos en Ciencias Sociales, 6(1), 43-72. Bogotá: Universidad Santo Tomás. DOI: https://doi.org/10.15332/ s2339-3688.2018.0001.02

1 Socióloga. Correo electrónico: luisafzamu@gmail.com 


\title{
RESUMEN
}

La habitabilidad en calle en Bogotá (Colombia) es un fenómeno social histórico. Al respecto se han planteado políticas basadas en el reconocimiento de los habitantes de calle como sujetos de derecho, sin embargo, a pesar de ese desarrollo normativo, este grupo poblacional ha sido víctima de violencias tanto por la sociedad civil como por el Estado. El objetivo de esta investigación es analizar la manera en que las políticas dirigidas a los habitantes de calle contribuyen a su desarrollo humano integral o transgreden su vida. La base teórica es la evolución del concepto de desarrollo humano integral. A través de un enfoque metodológico cualitativo se encontró como resultado que las políticas han contribuido al desarrollo humano integral únicamente de los habitantes de calle de los Centros de Atención, al mismo tiempo que se ejerce violencia contra las personas que han escogido la calle como proyecto de vida.

Palabras clave: habitabilidad en calle, violencia, desarrollo humano integral.

\begin{abstract}
Homelessness in Bogotá (Colombia) is a historical social phenomenon. To address this issue, policies has been formulated based on the acknowledgement of street inhabitants as legal subjects. However, despite this policy development, this population group has suffered violence perpetrated by both civil society and the State. The purpose of this research is to analyze the way in which policies for homelessness either contribute to their integral human development or transgress their life. The theoretical bases for analysis are the theory of human integral development, through a qualitative methodological approach. The results concluded that policy has contributed to integral human development only for homeless population that are already under the protection of an Attention Center, while simultaneously perpetrating violence against people who have chosen the streets as their life project.
\end{abstract}

Keywords: Homelessness, violence, integral human development. 


\section{INTRODUCCIÓN}

La habitabilidad en calle es un fenómeno social predominantemente urbano, asociado frecuentemente a la pobreza extrema y al escaso o nulo acceso de los ciudadanos a las oportunidades, derechos y beneficios de la ciudad, es decir, la negativa a garantizar las necesidades humanas básicas: la salud, la educación, la vivienda, la alimentación, la seguridad, el trabajo, el ocio, entre otros elementos que aseguran una óptima calidad de vida.

Ahora bien, Colombia, declarado un Estado social de derecho, establece en la Constitución Política de 1991 que es un país basado en principios y valores como la vida, la solidaridad, el respeto de la dignidad humana y la participación ciudadana. De acuerdo con esto, el Estado colombiano reconoce recientemente a los habitantes de calle como sujetos de derecho y por lo tanto elabora un conjunto normativo destacando la Ley 1641 de 2013 y el Decreto 560 de 2015.

A pesar de la existencia de esas normas, se evidencia que el pensamiento predominante en la sociedad es el rechazo hacia los habitantes de calle, el no reconocimiento de sus derechos como ciudadanos o la indolencia frente a su realidad. Pero es de mayor preocupación que la indiferencia y la violencia no sean actitudes propias de la sociedad civil, sino que también sean imperantes desde el mismo Estado, quien no reconoce -a pesar de ese desarrollo normativo- a los habitantes de calle como sujetos de derecho al no garantizar una vida digna para ellos y al invisibilizar sus problemáticas.

Las políticas públicas desarrolladas para habitantes de calle en Colombia son muy recientes, y aunque cada vez son más completas, tienen contradicciones en su visión de desarrollo humano integral. En ese sentido, la pregunta orientadora de esta investigación es, ¡cómo las políticas encargadas de la habitabilidad en calle han contribuido al desarrollo humano integral de los habitantes de calle o han vulnerado su calidad de vida desde el año 1997 al año 2017? 


\section{Metodología}

El enfoque metodológico de esta investigación es principalmente cualitativo, descriptivo y explicativo del paradigma histórico hermenéutico, debido a la consideración de la realidad social como múltiple y de los sujetos como portadores interactivos de significados susceptibles de analizar, haciendo énfasis en la comprensión de la experiencia y en la interpretación del recurso cultural desde Gadamer (1998), quien concibe la hermenéutica desde un punto de vista de la consciencia histórica, la autocrítica y de la experiencia de la verdad a través de una apropiación comprensiva de sentido, además determina que los hechos sociales son símbolos o textos que deben interpretarse y no solo describirse o explicarse.

Para contrastar la visión de desarrollo humano integral del Estado colombiano con los referentes teóricos ajustados a las condiciones de los habitantes de calle, se realizó una entrevista con Juanita Barrero, funcionaria del Ministerio de Salud, quien se encuentra participando en el equipo de profesionales que están construyendo la política pública nacional para habitantes de calle.

Se elaboró una revisión y análisis de fuentes secundarias: planes de desarrollo desde 1995 al 2016, informe especial sobre habitantes de calle "Bogotá cómo vamos", 6 censos de habitantes de calle en Bogotá (1997, 1999, 2001, 2004, 2007 y 2011), ya que la investigación se realizó hasta el 2016, el censo del año 2017 no está incluido, la Ley 1641 de 2013 y la política pública para habitantes de calle de Bogotá.

Finalmente, para confrontar las percepciones de las políticas dirigidas a habitantes de calle entre los expertos y los habitantes de calle, se pensaban realizar entrevistas con los funcionarios o directores de tres Centros de Atención (los expertos): Hogar de Paso Día-Noche Bakatá (fase 1), Centro de Atención Transitorio carrera 35 (fase 2) y Comunidad de Vida Hogar el Camino (fase 3), de los cuales solo se pudo efectuar con un funcionario del Centro de Atención fase 3, debido a que la Secretaría de Integración Social -SDIS- no otorgó una respuesta efectiva a la solicitud de permiso, también se tenía previsto el desarrollo de 3 grupos focales con los habitantes de calle de los mismos Centros de Atención, logrando solo uno en el Centro de Atención fase 3 con dos mujeres habitantes de calle gracias a un contacto personal. 


\section{INVESTIGACIONES ACERCA DE HABITABILIDAD EN CALLE}

Acerca de la habitabilidad en calle, son múltiples las investigaciones que se presentan desde distintas disciplinas, las cuales concluyen que la población habitante de calle ha sido violentada tanto física como simbólicamente, principalmente con marginación y exclusión social por parte de la sociedad civil, pero existe un vacío en cuestionarse la violencia hacia esta población por parte del Estado.

Entre las investigaciones latinoamericanas consultadas, las más relevantes son: "Personas en situación de calle: reconocimiento e identidad en contexto de exclusión social” (Weason, 2006), en los resultados de la investigación, se encuentra que el reconocimiento de los ciudadanos hacia los habitantes de la calle es fundamental en su construcción de identidad, ya que los legitima como miembros de la sociedad, mientras que el desconocimiento contribuye a que no sea posible la integración social, entre tanto, Navarro y Gaviria (2010) en la ciudad de Medellín evidencian las representaciones que la sociedad civil tiene sobre los habitantes de calle, obteniendo como resultado que la mayoría de las personas relacionan habitar en la calle como sinónimo de indigencia y de mendicidad, al considerar que se encuentran en esa situación debido a sus condiciones de pobreza; también se les reconoce como "locos" por sus facultades mentales y consecuencia del consumo de sustancias alucinógenas, incluso los ciudadanos que solo fijan su atención en la apariencia de los habitantes de calle, los denominan "desechables", despojándolos de su posición como personas y desconociendo que son una realidad cultural con su propia lógica de vida.

También declaran los autores que las personas tienen esa disposición hacia las poblaciones como consecuencia de su aspecto sucio y de actitud hostil, incluso por posibles experiencias de hurtos e incomodidades frente a la invasión del espacio público.

Por otro lado, Chaverra (2011) asegura que este no reconocimiento de los habitantes de calle como sujetos de derecho se debe a la insuficiencia del Estado para hacer llegar a esta población sus derechos y los medios para hacerlos efectivos, además explica que las políticas públicas no son opciones sino obligaciones del Estado para hacer más tolerable la vida en la calle. 


\section{Historia de la HABITABILIDAd EN CALle EN Bogotá}

Los primeros registros sobre habitabilidad en calle en la ciudad de Bogotá datan del siglo XIX, antes de eso se presentaban prácticas de mendicidad asistidas por instituciones particulares que dependían de la solidaridad de la comunidad (Política pública de habitantes de calle, 2015).

Durante los años 1970 y 1980, las migraciones aumentaron al igual que la idea de encontrar nuevas oportunidades de vida en la ciudad, lo que trajo consecuencias como el surgimiento de los caciques y jíbaros expendedores de droga, para lo cual, la solución de la Alcaldía fue la construcción de avenidas principales alrededor del sector de Santa Inés, aislando e intensificando las actividades ilegales (Alcaldía Mayor de Bogotá, 2010).

Desde 1998 hasta el año 2001, la Alcaldía de Enrique Peñalosa con su plan de desarrollo "Por la Bogotá que queremos", dio prioridad al mejoramiento de la calidad de vida de los habitantes de calle, mediante el aumento de la cobertura en acceso a servicios públicos básicos, principalmente en servicios de salud y educación primaria, así como también autorizó la expulsión de los habitantes de la calle y demolición del sector El Cartucho. Fueron demolidas 17 manzanas y se movilizaron los habitantes hacia otro sector como parte del programa de Renovación Urbana (Alcaldía Mayor de Bogotá, 2010). Mientras allí se construía el parque Tercer Milenio, los habitantes expulsados se trasladaron a una cuadra de la Dirección de Reclutamiento del Ejército y a dos de la Policía Judicial y del comando de la Policía Metropolitana, la denominada calle del Bronx, replicando las mismas problemáticas.

Debido a que este el fenómeno fue creciendo a gran escala en varias ciudades, alertó a las autoridades nacionales y motivó a que tomaran cartas en el asunto, reconociendo que era una problemática de orden nacional. Es por eso que se formula y todavía se encuentra en construcción la política pública nacional de habitantes de calle, Ley 1641 de 2013. El mayor avance en términos legales pertenece a la ciudad de Bogotá, que cuenta con su propia política pública para habitantes de calle, Decreto 560 de 2015.

El último hecho trascendental se presentó el 27 de mayo de 2016, cuando tuvo lugar en el centro de la ciudad de Bogotá el desalojo policial que se realizó en la calle del Bronx, ordenado nuevamente por el alcalde Enrique Peñalosa. La intervención en este sector del centro tuvo como objetivo desmantelar bandas delincuenciales que 
se dedicaban al expendio de drogas, secuestros y asesinatos, además de recuperar un espacio histórico y simbólico para la ciudad. Como consecuencia del operativo, los habitantes de la calle que allí residían se vieron en la obligación de trasladarse a otros sectores aledaños para asentarse, así lo mencionó el diario El Espectador (2016ª; $2016^{\text {b }}$ ) en varias ocasiones por las quejas de los residentes, comerciantes y los mismos habitantes de calle que aseguraban ser obligados a instalarse en determinadas calles y puentes.

\section{El DESARrollo hUMANO INTEGRAL DE LOS HABITANTES DE CALLE EN Colombia}

El Estado puede entonces fomentar la vida o la muerte de determinados grupos, y en ese sentido los impactos de la aplicación de determinadas políticas pueden oscilar entre la promoción del desarrollo humano integral o la muerte en virtud de que están excluidos. Se requiere, por lo tanto, hacer una revisión del concepto de desarrollo y de relacionarlas con el panorama latinoamericano, colombiano y bogotano. Una vez identificada la idea de desarrollo en la ciudad, es posible señalar aspectos como la visión de futuro y autosuficiencia para desarrollarse con sus propios recursos y las prioridades estatales.

A partir del año 1949, el presidente de los Estados Unidos Harry Truman mencionó públicamente el concepto de desarrollo como un modelo a seguir para todos los países del mundo. Es en este discurso que las clasificaciones entre países se hicieron aún más evidentes, declarándose unos como desarrollados y otros como subdesarrollados, debido a que las variables de esta dicotomía tenían relación principalmente con la pobreza (Múnera, 2007).

En 1969, Dudley Seers planteó que el desarrollo no podría medirse en términos económicos si cuestiones como el desempleo, la pobreza y la desigualdad aumentaban, a lo que en 1974 Hollis Burney Chenery agregó que debía tenerse en cuenta la distribución de la riqueza para asegurar el bienestar (Múnera, 2007).

En ese mismo año, entidades como el Banco Mundial (BM) y la Organización Internacional del Trabajo (OIT) comenzaron a entender que el capital económico 
no era el único referente para medir el desarrollo de los países, pues en el mundo se presentan territorios con alta generación de riqueza, pero concentrada en un grupo poblacional específico representado en una minoría, situación que amplía la brecha de desigualdad y no permite el alcance del desarrollo social y humano, es decir, la mayoría de las personas presentan necesidades básicas insatisfechas y por ende un bajo nivel de vida y bienestar (Urra, 2016).

Lo anterior, es consecuencia de la inefectividad de las autoridades para garantizar capacidades a las personas, las tres capacidades esenciales para un desarrollo humano satisfactorio consisten en que las personas puedan vivir una vida larga y saludable, alcancen altos niveles de instrucción y cuenten con los recursos necesarios para tener un nivel decente de vida, de no lograrse esas capacidades básicas, no se cuenta con las suficientes alternativas y oportunidades de realización (Groppa, 2004).

El Programa de las Naciones Unidas para el Medio Ambiente (PNUMA) y la Conferencia de Naciones Unidas sobre Comercio y Desarrollo (UNCTAD) en el simposio de Cocoyoc en 1974, afirmaron que cualquier proceso de crecimiento que no satisfaga o que obstaculice el acceso a la vivienda, el vestido, la salud y la educación, no es un proceso de desarrollo, además de mencionar que existen otras necesidades como la libertad de expresión, el derecho a manifestar o recibir ideas y a participar. Finalmente fue necesario entender que cada sociedad es endógena y decide sobre su propia visión de futuro y autosuficiencia, donde se sostienen las bases del desarrollo en sus propios recursos (Urra, 2016).

A partir de estos planteamientos, surgieron teorías y estudios realizados por varios economistas y profesionales en ciencias sociales, con el objetivo de definir en qué consiste el desarrollo social, de qué forma puede medirse y cómo alcanzar el desarrollo en los países que enfrentan grandes problemas sociales.

\section{LAS TEORÍAS DEL DESARROLLO SOCIAL}

Antes de teorizar sobre este concepto, es Manfred Max-Neef (1993) quien abre la puerta a lo que él denominó el "desarrollo a escala humana", reconociendo la 
importancia de pensar el desarrollo en relación con las comunidades e individuos y de definir y categorizar las necesidades de los seres humanos. La propuesta elaborada por Manfred Max-Neef (1993), economista chileno, considera 4 categorías existenciales (ser, estar, hacer y tener) y 9 categorías axiológicas (subsistencia, protección, afecto, entendimiento, participación, ocio, creación, identidad y libertad). Cada necesidad tiene una serie de satisfactores dependiendo del contexto (satisfactores singulares que solo cubren una necesidad, satisfactores destructores que al cubrir una necesidad imposibilitan otra, seudosatisfactores que generan una falsa satisfacción ilusoria en el tiempo, satisfactores inhibidores que satisfacen completamente una necesidad imposibilitando drásticamente otras y los satisfactores sinérgicos, que cubren una necesidad específica y al tiempo contribuyen a la satisfacción de otras necesidades).

Por lo cual el desarrollo consistiría en identificar necesidades, satisfactores, recursos y potencialidades, la falta de los dos últimos conlleva a la pobreza o distintas pobrezas no necesariamente económicas. La intención es que la comunidad proponga los satisfactores para el cubrimiento de las necesidades, así no son sujetos pasivos que reciben el desarrollo diseñado por los gobiernos, sino que se involucran en los procesos que conducen a él.

Mahbub Ul Haq (2003), un economista pakistaní, expresa que el primer elemento para tener en cuenta en el desarrollo de un país son las prioridades particulares elegidas por los gobernantes, por ejemplo, si un Estado prefiere hacer inversiones sociales o militares, si busca privilegiar de forma elitista o igualitaria, etc. Esto determina si la expansión del ingreso entre los habitantes funciona o no, debido a que en algunas sociedades el cubrimiento de una necesidad puede generar menos satisfacción que en otras, no se trata de la acumulación de la riqueza, sino de la distribución de esta a través de políticas públicas de los gobiernos en servicios sociales y políticas fiscales como son las reformas agrarias, sistemas de impuestos progresivos, sistemas de créditos, expansión de servicios sociales básicos, remoción de barreras para el ingreso de personas a la esfera económica y política, igualdad de oportunidades y redes de seguridad para personas ignoradas por el mercado o por las políticas públicas.

El desarrollo se entiende como el bienestar medido a través de indicadores sociales como el nivel de educación, salud, seguridad frente a crímenes y violencia física, tiempo libre satisfactorio, medioambiente limpio, libertades políticas y culturales, acceso a servicios básicos, igualdad, esperanza de vida, entre otros, ahora la 
generación de la riqueza ya no es un fin sino un medio para lograr cubrir las necesidades básicas de la población (Ul Haq, 2003).

Streeten, economista austriaco, menciona que entre las opciones que las personas tienen se incluyen la libertad política, social, económica y cultural, un sentido de comunidad, oportunidades de ser creadores y productivos, el respeto por sí mismo y los derechos humanos. Pero el desarrollo humano es mucho más que simplemente lograr esas capacidades; es también el proceso de procurarlas de manera equitativa, participativa, productiva y sostenible.

Debe destacarse la importancia del enfoque centrado en el ser humano, propuesto por Amartya Sen (2000). Para el economista indio, el desarrollo se centra en 4 grandes ejes: el ser humano como fin del desarrollo, ética, equidad y libertad de elección. En esta perspectiva, el desarrollo se crea desde el individuo, pues al tener distintas motivaciones se tienen distintos conceptos de bienestar, pero el elemento más importante radica en la posibilidad de que cada persona tenga la capacidad para conseguir las realizaciones que consideren valiosas. La ética funciona de la siguiente manera: las comunidades en general defienden sus intereses particulares por lo que el Estado responde a ellas directamente, la cuestión se presenta cuando entre comunidades se limita la cooperación, porque las políticas del Estado están enfocadas en el "yo" y no en el "nosotros", el nivel ético aparece aquí relacionado con el grado de satisfacción que tienen las personas al conocer la injusticia sobre los otros, lo que se plantea entonces, es que para lograr el desarrollo no siempre debe haber grupos sacrificados como se ha pensado, sino que se puede partir de una idea de cooperación para alcanzar el desarrollo. En cuanto a la equidad, Sen (2000) defiende una política de distribución de acceso a los servicios básicos para conseguir una igualdad real de derechos que mejoren la calidad de vida y permitan desarrollar las capacidades; y finalmente se plantea la libertad de elección de un proyecto de vida como fin último del desarrollo.

El Programa de las Naciones Unidas para el Desarrollo - PNUD, en el cual Sen fue uno de los grandes influyentes, entiende el desarrollo humano en la medida que exista un entorno en el que las personas pueden explotar su máximo potencial y llevar una vida creativa y productiva de acuerdo con sus necesidades e intereses, "las personas son la verdadera riqueza de las naciones" (PNUD, s.f.), el desarrollo implica ampliar las oportunidades para que cada persona pueda vivir una vida que valore. 
El ejercicio de las capacidades humanas se torna en el aspecto más importante para disfrutar una vida larga y saludable, un nivel de vida digno y participar en la sociedad, la finalidad del desarrollo es el bienestar humano y desde el punto de vista de los derechos humanos es la libertad para fortalecer capacidades y ejercer derechos, para hacer uso de sus alternativas y participar en la toma de decisiones que afectan sus vidas. A partir de estos postulados, el PNUD crea en 1989 la primera forma de medir el desarrollo humano, el Índice de Desarrollo Humano - IDH, que mide la esperanza de vida al nacer, la alfabetización en adultos y el PIB per cápita, se presenta anualmente para cada país y busca ser una herramienta de análisis político que refleja las prioridades de la gente, identifica desigualdades y mide el progreso (PNUD, s.f.).

\section{El desarrollo en América Latina}

Bernardo Kliksberg (2003), economista, sociólogo y contador público argentino, pretende demostrar por qué América Latina tiene los índices más altos de pobreza del mundo a pesar de tener una gran riqueza natural y social, además menciona que es posible considerar un territorio sin pobreza, porque la pobreza no es un hecho histórico, aunque cada día la medición cuantitativa señala que esta no habría dejado de ser significativa.

Según Kliksberg (2003), en América Latina se están presentando problemas sociales como el desempleo predominantemente en jóvenes, el déficit de educación, de salud, la desigualdad, la desnutrición y la explotación infantil, lo cual es contradictorio en lugares donde se establece la democracia, porque las personas inscritas en un régimen democrático exigirían que se destinaran los recursos a satisfacer esas necesidades.

Por otro lado, generalmente la pobreza también supone la desestabilización de la unidad familiar, lo que también influye en los índices de la violencia doméstica, el aumento de la criminalidad, a lo que las autoridades están respondiendo de forma punitiva, pero el camino de la punición no mejora la crisis social, porque la cárcel no ataca las causas estructurales de la criminalidad, con esto también toma auge la criminalización de la pobreza, lo que pasa de la solidaridad al aislamiento, la exclusión, la marginación y la represión (Kliksberg, 2003). 
En países desarrollados la prevención, la educación y la cultura son las bases de una sociedad más justa, entre mayor es el nivel de desarrollo humano, mayor es la preocupación por la autorrealización, el ambiente, la creatividad e independencia en el trabajo, la autonomía personal y la participación, además es mayor la confianza en la conducta de las otras personas (Kliksberg, 2003).

Existen barreras de pensamiento que no permiten que el desarrollo humano se alcance, en primer lugar, hay ciertos sectores de la población que justifican la pobreza como un problema individual, como si fuera una elección y tuviera que ver con la ignorancia, porque no les gusta trabajar, esto nos ha hecho pensar el biopoder tratado en el capítulo anterior, que es más fácil culpar a las personas que a los problemas estructurales que conducen al problema. La segunda idea es que el desarrollo con el paso del tiempo tendrá sus frutos y se acabará la pobreza, cuando no es así, el desarrollo ni siquiera alcanza a llegar a la clase media o hay que esperar hasta morir. La tercera idea es restarle importancia a la política social, que implica no pensar que los cambios en la mayor cobertura de los servicios básicos incrementan el desarrollo. Finalmente, la cuarta idea es que se pierda la capacidad de indignación de las personas frente a las injusticias sociales (Kliksberg, 2003).

Valverde (2015) menciona que los Estados han reconocido desde hace mucho tiempo que la pobreza es una condición humana compleja, que se caracteriza por la privación continua o extrema de los recursos, la capacidad, las opciones, la seguridad y el poder necesarios para disfrutar de un nivel de vida adecuado y de otros derechos civiles, culturales, económicos, políticos y sociales. Por lo tanto, deben adoptar medidas y políticas de amplio alcance para eliminar las condiciones que causan la pobreza, la acentúan o la perpetúan, y asegurar la realización de todos los derechos económicos, sociales, culturales, civiles y políticos de quienes viven en la pobreza.

Los gobernantes de los países exitosos dicen que el desarrollo social es motor del desarrollo económico, "el Estado debe ser responsable por necesidades básicas como la salud y la educación, eso es irrenunciable, forma parte de las constituciones y del credo ético de nuestras sociedades" (Kliksberg, 2003, p. 11). Además, indica que en el modelo de desarrollo integrado las personas participan, es decir, no son pasivas, sino que se articulan y se movilizan. 


\begin{abstract}
Algunas de las políticas que se han implementado en los países en vías de desarrollo son: programas focalizados en los intereses de grupos vulnerables en vez de la industrialización, también lograr bajar los precios y las tasas de interés, reducir las importaciones, la descentralización y otorgarles la misma importancia a las organizaciones sociales que a las burocráticas (Kliksberg, 2003).
\end{abstract}

\title{
Desarrollo humano integral en Colombia
}

Posteriormente María Cecilia Múnera (2011), colombiana y licenciada en educación, se refiere al desarrollo como una construcción sociocultural múltiple, histórica y socialmente determinada, en donde cada grupo social establece sus finalidades fundamentadas en sentidos de vida que se construyen colectivamente, las características son:

El ser humano se considera sujeto de desarrollo cuando puede ponerse en el centro de su propio mundo, conocer su pasado, identificar su presente y visualizar su futuro afirmando su libertad y construyendo su propia identidad, es decir, buscar un horizonte de vida, ser consciente de su existencia y dotarla de sentido. La naturaleza es un sistema con el que se interactúa, el hábitat es el ámbito de realización de los sujetos, el lugar de partida y de realización de múltiples proyectos sociales (Múnera, 2011).

La democracia debe estar fundamentada por valores y no por normas, en donde se garantice autonomía política. Se trata de un desarrollo sinérgico y toma en cuenta la historia, las tradiciones y los recursos particulares de cada comunidad para la resolución de conflictos y satisfacción de necesidades, autogobierno como proyecto político autosustentable (Múnera y Sánchez, 2012).

La participación es un proceso permanente de construcción sociocultural, es la base del desarrollo, en el tipo de participación sinérgica, los sujetos muestran una predisposición positiva y de credibilidad crítica para comprometerse con el proceso de desarrollo, teniendo motivaciones e incentivos de forma personal, social y humanitaria, por lo que parte de intereses comunes y construyen tanto su propia identidad como la colectiva, se favorece la tolerancia, el respeto por la diferencia, la 
honestidad y la ética generando confianza y sentido de pertenencia, y se estimulan las potencialidades de los sujetos para darle continuidad y sostenibilidad (Múnera, 2011).

El desarrollo no restringe, por el contrario, supone que en la diversidad se encuentra gran parte de la riqueza de la participación. El desarrollo debe evidenciarse en el sistema político, sistema social y sistema económico. Si bien la participación del Estado afecta, no debe ser el centro del potencial del desarrollo (Múnera, 2011).

Con este barrido conceptual, se evidencia que el desarrollo ha sufrido transformaciones que cada vez se centra más en el individuo e involucran diferentes variables que superan la visión economicista, formando lo que Múnera ha decidido nombrar como desarrollo humano integral, con este proceso también han evolucionado las formas de medición de dicho desarrollo, sumando variables cualitativas.

Estas mediciones propuestas, sin embargo, no son aplicables a todas las comunidades y poblaciones, debido a que en gran medida se sigue pensando en un estilo de vida generalizado, por lo tanto, para poder llegar a una población particular como son los habitantes de calle, es necesario retomar las variables que tienen en cuenta su forma de vida.

Los ciudadanos habitantes de calle son sujetos históricamente situados, y más allá de las condiciones materiales, habitar la calle implica la constitución de su propio entorno económico, físico, social, cultural y ambiental, donde se construyen identidades, formas de ser, de pensar, de sentir, de hablar, así como conjuntos de normas sociales, valores y principios bajo los cuales hacen una lectura de la realidad, tal como lo plantea la Secretaría Distrital de la Mujer, además menciona que la complejidad del fenómeno implica:

Comprender la calle como un espacio social y cultural opcional para un grupo considerable de personas no necesariamente en condición de pobreza como sugieren las visiones tradicionales de las y los sin techo, sino atraídas y obligadas por distintas situaciones que subyacen en lo estructural y donde es posible conformar afectos, roles, rutinas, identidades y hasta lenguajes propios por fuera del ordenamiento clásico de la sociedad. (Grajales, 2015, p. 59) 
Por lo tanto, para esta investigación, se propone medir el desarrollo humano integral de los habitantes de calle con la siguiente matriz de variables:

Tabla 1. Matriz DHI habitantes de calle

Matriz de desarrollo humano integral para habitantes de calle

\begin{tabular}{|c|c|}
\hline \multirow[t]{4}{*}{ ONU } & Salud \\
\hline & Educación \\
\hline & Libertad de expresión \\
\hline & Participación \\
\hline \multirow{2}{*}{ Mahbub Ul Haq } & Seguridad \\
\hline & Tiempo libre satisfactorio \\
\hline \multirow[t]{6}{*}{ Paul Streeten } & Libertades políticas \\
\hline & Libertades económicas \\
\hline & Libertades culturales \\
\hline & Sentido de comunidad \\
\hline & Oportunidad de ser creador o productivo \\
\hline & Respeto por sí mismo \\
\hline \multirow[t]{2}{*}{ Amartya Sen } & Capacidades humanas \\
\hline & Proyecto de vida \\
\hline \multirow[t]{5}{*}{ Max Neef } & Subsistencia \\
\hline & Afecto \\
\hline & Entendimiento \\
\hline & Identidad \\
\hline & Libertad de elección \\
\hline \multirow[t]{2}{*}{ Bernardo Kliksberg } & Autorrealización \\
\hline & Autonomía personal \\
\hline \multirow{2}{*}{$\begin{array}{l}\text { María Cecilia } \\
\text { Múnera }\end{array}$} & Dignidad humana \\
\hline & $\begin{array}{l}\text { Pertenencia simultánea a múltiples grupos de } \\
\text { forma integral }\end{array}$ \\
\hline
\end{tabular}

Fuente. Elaboración propia

Esta investigación tomará como concepto base de desarrollo humano integral a María Cecilia Múnera (2011), la cual menciona que el desarrollo radica en la realización de los sujetos como seres individuales y colectivos, construyendo proyectos en común y perteneciendo simultáneamente a múltiples grupos de forma integral (físico, biológico, social, político, económico, afectivo y espiritual), su estrategia es 
configurar sujetos sociales a partir de proyectos educativos múltiples y dinamizar los procesos sociales incluyentes.

También teniendo en cuenta a la política pública de habitantes de calle de Bogotá y el componente de Desarrollo Humano Integral, entendido como el desarrollo de capacidades y la ampliación de oportunidades para alcanzar la inclusión social y la realización de un proyecto de vida, en el caso de los habitantes de calle se constituye en: libertades negativas como estar libre de violencia, de enfermedades y en libertades positivas como ser y hacer, lo que considera valioso para su vida para conocer desde un enfoque diferencial las necesidades y potencialidades de los habitantes de calle.

Para la Política Nacional de habitantes de calle, la concepción de desarrollo humano integral en este caso se toma desde la Agenda para Colombia 2030 de las Naciones Unidas, entonces el desarrollo humano integral es acogido como el fomento de las capacidades de las personas para que puedan tener oportunidades y libertad de autodeterminación de su curso de vida, de su historia de vida.

Las concepciones de ambas Políticas Públicas son similares y tienen como base fundamental el desarrollo humano integral, además se asocian a los postulados de Amartya Sen, sin embargo, deja pasar varios factores que influyen en el desarrollo humano integral. En el siguiente apartado surgen unas tensiones que pretenden demostrar que hay un avance en la definición de habitabilidad en calle y del concepto de desarrollo en las personas que habitan la calle, aún hacen falta acciones que garanticen a todo este grupo la posibilidad de alcanzar un desarrollo humano integral en los términos planteados según la matriz propuesta. En ese sentido, el objetivo es entonces conocer el alcance de las políticas dirigidas a los habitantes de calle a la luz de estas propuestas.

\section{Resultados}

En primer lugar, se partirá de una clara diferencia entre las personas que han escogido la calle como opción de vida y las personas que han llegado a esta condición por 
diversos factores, por esta razón, el Estado no puede actuar de la misma forma ante ambos casos, y aunque hasta el momento esa distinción no se ha tenido en cuenta, se discute para la futura política pública nacional.

Es importante tener una postura crítica frente al postulado de las personas que escogen libremente la calle como opción de vida, ya que la cuestión principal es, ¿qué condiciones conducen a los sujetos a hacer voluntariamente de la calle su lugar de habitación? Pues podría deducirse que esta situación se presenta como consecuencia de las estructuras sociales que se han legitimado, como reconoce el Estado, y que encontramos un sistema excluyente de los privilegios de tener una óptima calidad de vida, lo que ocasiona que las personas normalicen el hecho de vivir en las calles; o se puede ir al hecho de considerar que las personas han tenido a lo largo de su vida situaciones tan problemáticas en su círculo social, que prefieren vivir en las calles porque han encontrado en este lugar cierta "libertad" y consideran que regresar a la vida de un ciudadano ordinario es un acto de regresión.

Por esto, afirma Juanita Barrero en la entrevista, que, si una persona decide que quiere una perspectiva distinta a vivir en la calle, el Estado debe garantizarle que eso sea posible y eso significa posibilitar que desarrollen las competencias necesarias que les van a permitir tener una inclusión social adecuada, y eso implica tener un primer nivel de la atención asistencial, para que en un principio la persona no continúe viviendo en la calle. Para que la persona logre una inclusión social real, hay que cambiar su estilo de vida mejorando las condiciones para obtener una buena calidad de vida y garantía de servicios y bienes que les permitan mantener su estilo de vida, de lo contrario la inclusión social no va a ser posible y no será posible salir del asistencialismo.

Por otro lado, en Colombia existe el derecho al libre desarrollo de la personalidad, eso quiere decir que las personas pueden escoger el estilo de vida que prefieran, "lo importante es que sea una escogencia, no que las condiciones lleven a optar por eso, o sea, no es que se esté viviendo así a pesar de lo que realmente se quiere" (Barrero, comunicación personal, 2017), pero sí existen personas que quieren vivir en la calle, tienen el derecho de hacerlo y el Estado debe garantizar que se pueda tener ese estilo de vida en condiciones de dignidad, y ahí es donde viene la asistencia. 
Si la persona quiere seguir viviendo en la calle, la asistencia significa un nivel distinto de dignidad, una de las variables mencionadas en la matriz de desarrollo, porque puede asearse, alimentarse, dormir, entre otras cosas. En palabras de una habitante de calle que hizo parte del grupo focal, inscrita hace dos meses en el proceso de uno de los Centros, "hay personas que no dejan la calle porque no se imaginan su vida de otra forma y no son capaces de cambiar su "cambuche" por una cama, además de que la calle les da dinero" (Grupo focal, habitante de calle, comunicación personal, 2017).

Figura 1. Variables del desarrollo humano integral

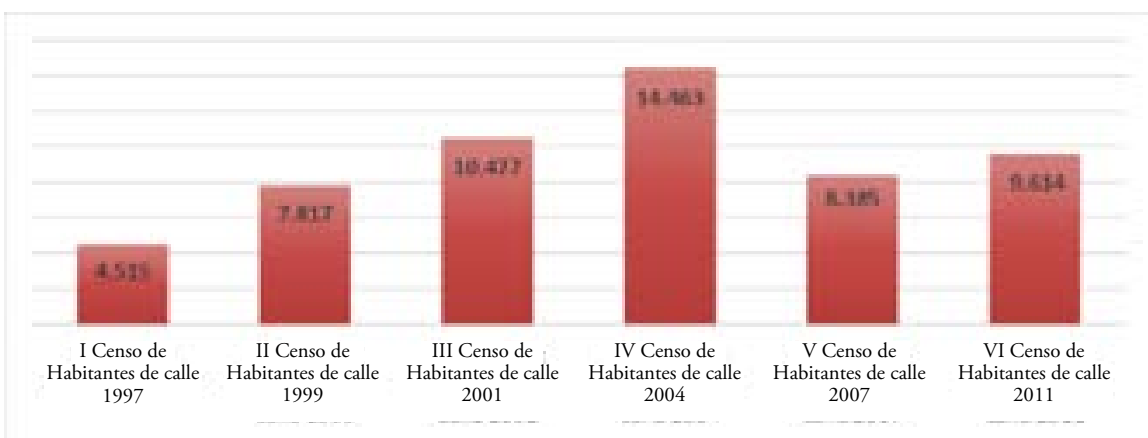

Fuente. Elaboración propia con base en los censos de habitantes de calle de Bogotá

Los censos realizados en Bogotá permiten tener, en primer lugar, una aproximación a las características de los habitantes de calle en la ciudad. La figura 1 presenta información sobre el número de habitantes de calle censados. Si bien se aprecia un incremento de la población debe relativizarse esa conclusión, ya que el método de recolección de la información influye en este resultado. La aplicación de encuestas debe enfrentarse al problema de estimación del universo de la población que se está tratando de describir. Al no existir ese dato es muy difícil establecer si el número de quienes respondieron el cuestionario corresponde al total o a una fracción de dicha población. Otro aspecto que hace difícil la comparación es que los censos de 2001 y 2004 recogen información de Bogotá y Soacha, mientras que los restantes solo de la primera. 
Figura 2. Sexo 1997-2011

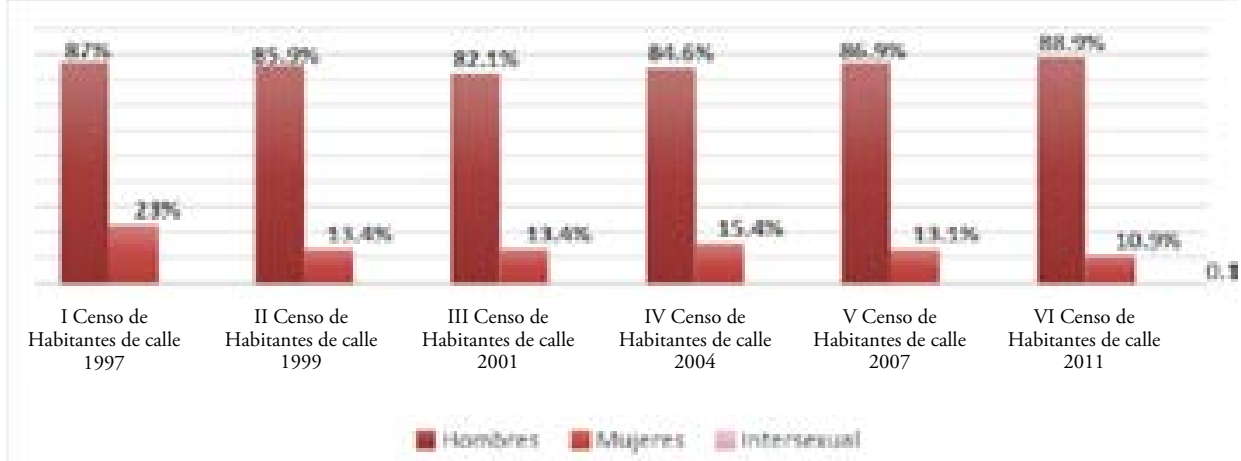

Fuente. Elaboración propia con base en los censos de habitantes de calle en Bogotá

En cuanto al sexo, las mujeres siempre han sido una minoría en la población habitante de calle y disminuyen con el paso del tiempo, mientras que los hombres habitantes de calle están cada vez más cerca del $90 \%$, el $0.2 \%$ es intersexual, cifra que se ha mantenido constante en el periodo 1997-2011. Debe agregarse que en el último censo se preguntó por el género, en este aspecto los resultados fueron que un $85.6 \%$ se afirmaba masculino, $10.7 \%$ femenino y 0.6 transgénero. Finalmente, en ese mismo censo se preguntó por la orientación sexual, obteniendo que el $98.4 \%$ es heterosexual, $1.1 \%$ homosexual y $1 \%$ bisexual.

A través de la revisión de autores que posibilitó la construcción de la propuesta de matriz ajustada a los habitantes de calle, es posible ver que siete de las variables consideradas (afecto, educación, salud, subsistencia, tiempo libre satisfactorio, capacidades y violencia, esta última analizada en un acápite posterior) se encuentran en estos censos y se pueden analizar a la luz de las teorías, también contienen otras variables susceptibles del análisis. 


\section{Afecto}

Figura 3. Apoyo familiar 1997-2011

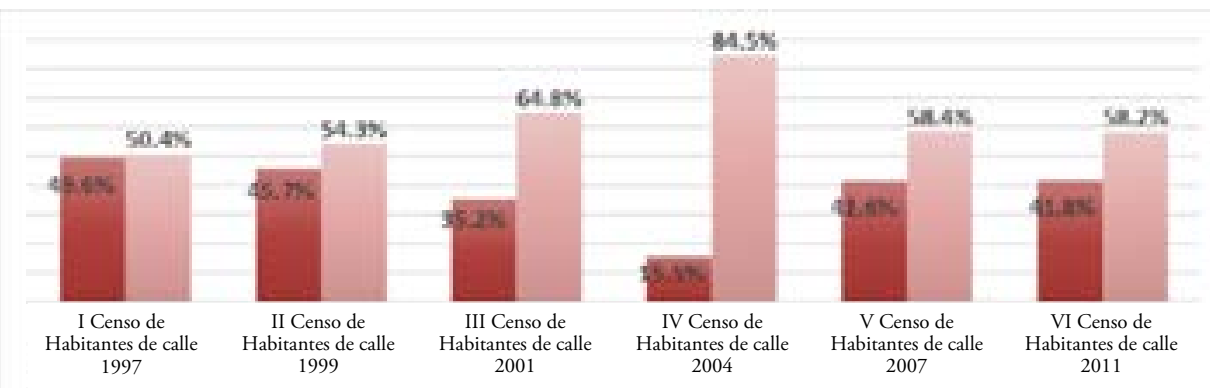

Fuente. Elaboración propia con base en los censos de habitantes de calle de Bogotá

La primera de ellas corresponde a la variable afecto, retomada desde Max-Neef, y en el censo remite como apoyo familiar, encontrando como resultado que los cambios más drásticos se presentan en el año 2001 y 2004. En ese periodo hay una fuerte disminución de apoyo, mientras que en los demás años se ha mantenido una constante cerca del $50 \%$ que sí los ayudan, y $50 \%$ que no, con tendencia a la disminución, en el año 1997 la red de apoyo se encontraba por encima del 50 \% y en el 2011 ya se encontraba por abajo. De las personas que afirman haber recibido apoyo, la mayoría es por parte de la madre, seguido de amigos, hermanos y demás familiares, e incluso no familiares, dejando como última cifra al padre.

\section{EdUCACIÓN}

La segunda variable involucrada en los censos de habitantes de calle es la educación, concepto retomado desde la propuesta de la ONU. En términos generales, se puede apreciar de la información reportada en los censos que predomina la situación de individuos con algún grado de estudios. Si bien en el censo de 1997 se registró el $67.1 \%$ sin estudio, esa cifra de personas no escolarizadas descendió para 1999 a 10.8 
$\%$ y se ha mantenido cerca de este porcentaje. Lo anterior significa que la educación como factor causal de la habitabilidad en calle ha tenido una evolución importante, por el contrario, desde 1999 aparece una minoría de habitantes de calle que aseguran tener algún grado de educación superior, la mayoría sin embargo, tiene algún año de la primaria cursado completamente.

\section{SALUd}

La variable de salud también propuesta por la ONU, es retomada en los censos de habitantes de calle, únicamente por el tema del consumo de sustancias psicoactivas (SPA), en ese caso los resultados fueron: con cifras superiores al $50 \%$, el bazuco es la sustancia psicoactiva más utilizada por los habitantes de calle, seguido de la marihuana y el alcohol. Es importante aclarar que las personas que declaran no consumir ninguna sustancia sicoactiva van decreciendo desde un $15.3 \%$ en el 2004, hasta un $5 \%$ en el 2011.

\section{SubSISTENCIA}

Subsistencia, propuesto desde Max-Neef, también es una variable que se puede evidenciar en los censos de habitantes de calle, reflejando que la principal actividad económica a la que se dedican los habitantes de calle es al reciclaje en casi un $70 \%$, el resto corresponde a mendicidad o trabajar limpiando vidrios, tocando llantas, entre otros.

\section{TIEMPO LIBRE SATISFACTORIO}

En cuanto al tiempo libre satisfactorio, variable propuesta por Ul Haq, habitualmente se dedican a escuchar música, ver televisión, leer, jugar o hacer ejercicios físicos, esta pregunta solo se tuvo en cuenta para el censo de habitantes de calle del año 2007. 


\section{Capacidades}

También podría decirse que hace referencia a la variable de capacidades, propuesta por Amartya Sen, al preguntarse por los talentos de los habitantes de calle, obteniendo como resultado en el censo de habitantes de calle del ańo 2007, que el $46.8 \%$ dice no tener ningún talento y el $52.5 \%$ afirma tener alguno, en su gran mayoría las artes, seguido de las lenguas, los deportes, la literatura, entre otros.

Finalmente, las variables que conllevan a que los habitantes de calle tengan una vida digna se solventan con apoyo del Estado, por eso también es de gran importancia conocer el comportamiento del papel gubernamental en estos años.

Figura 4. Apoyo institucional 1999-2011

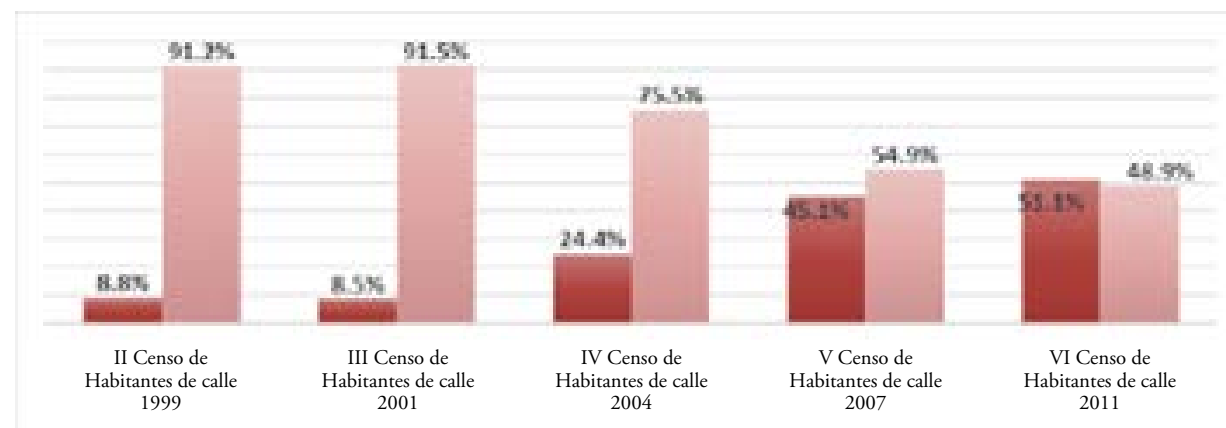

Fuente. Elaboración propia con base en los censos de habitantes de calle de Bogotá

Del acceso a servicios de apoyo institucional no se tienen cifras en el año 1997, pero en el año 1999 y 2001 es inferior al $10 \%$, es a partir del año 2004 que las cifras van aumentando considerablemente y a escalas grandes, registrando en 2007 y 2011 una situación pareja cercana al $50 \% / 50 \%$, siendo importante mencionar que hasta el año 2007 se preguntaba si recibía un apoyo institucional, mientras que en el año 2011 se preguntó si conocían los servicios de la SDIS y si aprovechaban los servicios o no, es decir, se tiene una visión más autónoma de los habitantes de calle al decir que los Centros de servicio están disponibles, y solo es decisión de los habitantes utilizar 
los servicios o no. En el año 2002, se atendió el 64.6 \% de la población habitante de calle en los hogares de paso, en el 2003 fueron $74.1 \%$, en el 2004 fue $48.4 \%$, en el 2005 fue $43.9 \%$, en el 2006 fueron $53.3 \%$, en el 2007 fueron $79 \%$, en el 2008 fue $75 \%$, en el 2009 fue $83.1 \%$ y en el año 2010 se atendió al $99.9 \%$ de los habitantes de calle, según el plan de desarrollo de Bogotá 2008-2012. En estas cifras se cuentan las personas que alguna vez han pasado por un Centro de atención, así sea de forma transitoria, al menos una noche, pero esto no garantiza algún seguimiento o que se hayan adoptado medidas para mejorar su calidad de vida.

Se reconoce que no se pudo completar la matriz de desarrollo humano a cabalidad, debido a que el tiempo de aplicación de los instrumentos de investigación fue insuficiente, por otro lado, no se permitió el abordaje sobre los temas de política y violencia, por falta del permiso que no otorgó la SDIS, además los censos no incluyen esas variables, solo los últimos, y aun así se sigue abordando de manera superficial. Quedan pendientes por analizar las siguientes variables: libertad de expresión, participación, libertades políticas, libertades económicas, libertades culturales, sentido de comunidad, respeto por sí mismo, proyecto de vida, entendimiento, identidad, libertad de elección, autorrealización, autonomía personal y pertenencia simultánea a múltiples grupos de forma integral.

Tendría que realizarse una investigación con mayor apoyo de la SDIS que permitiera completar todas las variables de la matriz, también sería pertinente buscar la forma de aplicarla a los habitantes de calle en calle, y hacer uso de otras estrategias diferentes a las entrevistas y las encuestas, que permitan conocer los resultados de variables más personales, como por ejemplo el "respeto por sí mismo" que puede llegar a ser ambiguo.

\section{SEGURIDAD}

En palabras de Carlos, el funcionario del Centro de Atención Comunidad de Vida el Camino, no hay acciones institucionales sobre las personas que se niegan a recibir el servicio. Lo anterior implica, según Juanita Barrero, que no se pueda garantizar la 
seguridad (una de las variables de la matriz de desarrollo humano) de los habitantes de calle en la calle y ellos lo saben, lo que convierte al proceso asistencial en la única alternativa para que muchas situaciones de violencia no se presenten. La figura 5 , por ejemplo, permite apreciar el tipo de violencias que han sufrido los habitantes de calle. La información recopilada en el censo realizado en 2011, señala como principal manifestación de violencia en contra de esta población las golpizas y los hurtos. En términos estadísticos, se evidencia la magnitud de esta problemática:

Figura 5. Violencia que han sufrido 2007-2011

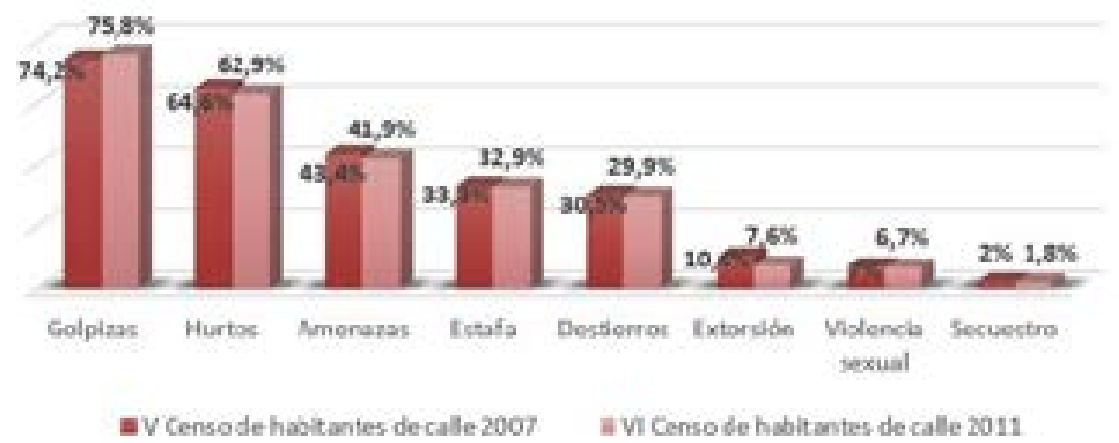

Fuente. Elaboración propia con base en los Censos de Habitantes de Calle 2007 y 2011

Para la variable de seguridad, se concluyó que la mayoría de los habitantes de calle aseguran haber sido víctimas de estos hechos delictivos, principalmente en un 51.4 $\%$ por la Policía y $24.4 \%$ por otros habitantes de calle, según los censos de habitantes de calle de Bogotá de los años 2007 y 2011.

Durante el año 2010 se presentaron 59 homicidios de habitantes de calle, en el 2011 fueron 92 casos de homicidio, en el 2012 se reconocieron 55 asesinatos, en el 2013 54, en el año 2014 se registraron 61 casos y en el año 2015 las víctimas de homicidio fueron 88 , de los cuales el $76 \%$ tenían de 21 a 40 años de edad, y el $11 \%$ entre los 11 y los 20 años de edad. Lo que quiere decir que en el año 2015 se incrementó en un $44 \%$, según datos de la DIJIN (Bogotá Cómo Vamos, 2016, Boletín especial habitantes de calle en Bogotá). 
En el primer semestre del año 2016 se presentaron 45 homicidios, el 80 \% estaban entre los 30 y los 39 años, según el informe especial de habitantes de calle de Bogotá Cómo Vamos, esto teniendo en cuenta que la seguridad humana y convivencia ciudadana era uno de los componentes de la política pública de habitantes de calle en Bogotá realizada en el 2015.

Las habitantes de calle del grupo focal de la Comunidad de Vida El Camino, no se explican cómo el Gobierno dejó pasar por tantos años los delitos de la calle del Bronx, según ellas, los policías tenían conocimiento de secuestros, prostitución, asesinatos.

\begin{abstract}
Entonces, eso es una crueldad que Colombia y la ciudad se haya tomado el Bronx hasta ahora y años atrás no se dieron cuenta, toda la cantidad de mujeres y nińos que se prostituyeron y toda la cantidad de personas que quedaron muertas ahí en el Bronx... Entonces, si no es otro extranjero, otra persona de otro lado que le pasa algo aquí, la ciudad se sigue perdiendo. (Grupo focal, habitante de calle en Centro de Atención Comunidad de vida hogar el camino, comunicación personal, 2017)
\end{abstract}

\title{
Conclusiones
}

Esta investigación encontró su límite al poner en evidencia las tensiones que se presentan en el ejercicio del poder político sobre una población históricamente compleja, y concluye que el tratamiento institucional que ha regulado la habitabilidad en calle ha contribuido en los últimos años a variables del desarrollo humano integral como la salud, la educación, el tiempo libre satisfactorio, la oportunidad de ser creador o productivo, fortalecimiento de capacidades, subsistencia, afecto y dignidad solo para aquellas personas que tienen la voluntad de inscribirse en un Centro de Atención y comenzar un proceso de inclusión a la sociedad civil, pero aún no se han encontrado los mecanismos necesarios para hacer posible y real el desarrollo humano integral de quienes han decidido optar por la habitabilidad en calle como proyecto de vida, por el contrario, se perpetúan ideas de violencia sutil contra ellas por parte de algunos funcionarios públicos y de la sociedad civil. 
Cabe aclarar que esta matriz se construyó con fines académicos de presentar un modelo de desarrollo ajustado a los habitantes de calle, pero sería inapropiado afirmar que los habitantes de calle que no tienen acceso a estas variables necesariamente están en una condición de subdesarrollados, pues el desarrollo debe ir ligado al proyecto de vida de las personas, y si por ejemplo un habitante de calle considera que para él no es importante educarse formalmente en una institución, pues no se está condenando al subdesarrollo, por eso no se incluye la variable vivienda desde la matriz de $\mathrm{DIH}$, porque si una persona prefiere dormir al aire libre con sus mascotas y ver el cielo descubierto, en vez de tener una casa lujosa, lo puede hacer de igual forma. Hay privación del desarrollo cuando no se tiene acceso y no se garantiza el acercamiento a estas variables, pese a que sí hacen parte del propósito de vida de la persona y lo considera fundamental para su vida.

La visión del DHI en Colombia está basado en el desarrollo de capacidades y ampliación de oportunidades para lograr la inclusión social y la realización de un proyecto de vida (libertades positivas y negativas), dejando de lado otras dimensiones: físico, biológico, social, político, económico, afectivo y espiritual.

Se puede entonces concluir que existe un interés del gobierno distrital por mejorar las condiciones de vida de esta población, pero entre las principales dificultades está la ausencia de una clara distinción entre las razones que explican la generación de esa situación de habitabilidad en calle, es decir, no distingue si las personas han llegado a esta condición por diversos factores estructurales o si es habitante de calle por voluntad propia.

Otro aspecto preocupante es que el Estado no cuente con la capacidad para garantizar las condiciones mínimas de vida digna para quienes no se vinculan a ningún programa, a los que a pesar de no ser un espacio habitable, deciden habitar la calle asumiendo una vida de riesgos.

En ese sentido, no se ejercen acciones institucionales para personas que han acogido la calle como proyecto de vida, y como consecuencia de esto hay altos índices de violencia, tanto por ellos mismos como por funcionarios públicos, los mismos que 
han sido cómplices en varias oportunidades de hechos delictivos. A pesar de que la seguridad es un componente de la política pública de habitantes de calle en Bogotá, los únicos espacios en que se garantiza una seguridad son los Centros de Atención, según los funcionarios.

Se presenta segregación territorial en la ciudad de Bogotá, se configuran espacios de rechazo y espacios de reconocimiento de los habitantes de calle y el gobierno ha potenciado esta situación generando aislamiento con construcciones o desalojos de calles.

Finalmente, este artículo pone en evidencia una incógnita que pretende ser abordada en posteriores investigaciones. Se sabe que se presenta violencia, tanto física como simbólica en el tratamiento institucional de los habitantes de calle que han escogido la calle como proyecto de vida, pero debido a las dificultades para realizar un trabajo de campo más profundo, queda pendiente averiguar, ¿en los Centros de Atención de la SDIS también se ejerce violencia contra los habitantes de calle? Pues se concluye también que el mismo gobierno está cuestionando los Centros de Atención como medio efectivo de inclusión social, se realizan exigencias que no son factibles para los habitantes de calle, como la desintoxicación, y habría que tener un mejor análisis de las prácticas que se realizan al interior de estas instituciones y cuestionar si los servicios se orientan a mantener la autonomía de las personas.

\section{REFERENCIAS}

Alcaldía Mayor de Bogotá, Secretaría Distrital de Integración Social. (2010). El Cartucho: del barrio Santa Inés al callejón de la muerte. Recuperado de http://old.integracionsocial.gov. co/anexos/documentos/aseptiembre/EL\%20CARTUCHO.pdf

Bogotá Cómo Vamos. (2016). Boletín especial cifra habitantes de calle en Bogotá. Recuperado de http://www.bogotacomovamos.org/documentos/cifras-habitantes-de-calle-en-bogota/

Chaverra, C. (2011). El habitante de la calle como sujeto de derecho. Revista Cultura Investigativa, (2). Medellín: Facultad de Derecho de la Universidad Santo Tomás. 
Recuperado de http://revistaci.blogspot.com.co/2012/07/el-habitante-de-la-calle-comosujeto-de.html

El Espectador. (2016a). Después del Bronx. Bogotá. Recuperado de https://www.elespectador. com/opinion/editorial/despues-del-bronx-articulo-649808

El Espectador. (2016). ¿Qué dejó la intervención del Bronx después de seis meses? Bogotá. Recuperado de https://www.elespectador.com/noticias/bogota/ dejo-intervencion-del-bronx-despues-de-seis-meses-articulo-667944

Gadamer, H. (1998). Verdad y método II, la universalidad del problema hermenéutico. Salamanca: Ediciones Sígueme S. A. Recuperado de http://medicinayarte.com/img/ gadamer-verdad_y_metodo_ii.pdf

Groppa, O. (2004). Las necesidades humanas y su determinación. Instituto para la integración de saber, Universidad Católica de Argentina.

Kliksberg, B. (2003). ¿Es posible construir una economía con rostro humano? Revista del CLADReforma y Democracia. Caracas. Recuperado de http://siare.clad.org/ revistas/0045800.pdf

Ley 1641. (Julio 12 de 2013). Por la cual se establecen los lineamientos para la formulación de la politica pública social para habitantes de la calle y se dictan otras disposiciones. Diario Oficial n. ${ }^{\circ}$ 48849. Recuperado de http://wsp.presidencia.gov.co/Normativa/Leyes/ Documents/2013/LEY\%201641\%20DEL\%2012\%20DE\%20JULIO\%20DE\%20 2013.pdf

Múnera M. (2007). Resignificar el desarrollo. Medellín: Facultad de Arquitectura de la Universidad Nacional de Colombia. Recuperado de http://www.bdigital.unal.edu. co/10438/1/32515305.2007.pdf

Múnera, M. (2011). El desarrollo entendido como construcción sociocultural múltiple, un enfoque desde el ser humano. Medellín, Colombia. Recuperado de http://www. comfenalcoantioquia.com/Portals/descargables/pdf/memorias_foro_edu/Dia1/EL_ DESARROLLO_COMO_CONSTRUCCI\%C3\%93N_M\%C3\%9ALTIPLE.pdf 
Múnera, M., y Sánchez L. (2012). Construcción social de hábitat: reflexiones sobre politicas de vivienda en Colombia. Quito, Ecuador: FLACSO - CLACSO. Recuperado de http:// biblioteca.clacso.edu.ar/clacso/gt/20120413125417/gthi1-3.pdf

Navarro, O., y Gaviria, M. (2010). Representaciones sociales del habitante de la calle. Universitas Psychologica, 9(2), 345-355. Recuperado de http://www.scielo.org.co/pdf/ rups/v9n2/v9n2a04.pdf

Neef, M. (1993). Desarrollo a escala humana: conceptos, aplicaciones y algunas reflexiones. Montevideo, Uruguay: Editorial Nordan-Comunidad.

PNUD. (s.f.). Reafirmación del desarrollo humano. Teoría desarrollo humano. Recuperado de http://www.dhl.hegoa.ehu.es/ficheros/0000/0570/Teoria_Desarrollo_Humano._ PNUD.pdf

Secretaría Distrital de Integración Social. (2012). Subdirección para la adultez, VI Censo Habitantes de Calle, Bogotá. Recuperado de https://www.minsalud.gov.co/sites/rid/Lists/ BibliotecaDigital/RIDE/INEC/IGUB/censo-habitante-calle-bogota-2011.pdf

Secretaría Distrital de Integración Social. (2016). Prevención y atención integral del fenómeno de habitabilidad en calle. Recuperado de http://old.integracionsocial.gov.co/anexos/ documentos/2016documentos/05082016_Prevencion_y_Atencion_Integral_del_ Fenomeno_de_Habitabilidad_en_Calle.pdf

Sen, A. (2000). Desarrollo y libertad. Barcelona: Ediciones Planeta.

Ul Haq, M. (2003). El paradigma del desarrollo humano. Recuperado de http://www. otrodesarrollo.com/desarrollohumano/MulHaqParadigmaDesarrolloHumano.pdf

Urra, M. (2018). Paradigma de desarrollo social y humano. Orígenes y pioneros. Recuperado de https://osf.io/preprints/socarxiv/ntfer.

Valverde, C. (2015). De la necropolitica neoliberal a la empatía radical: violencia discreta, cuerpos excluidos y repolitización. Barcelona: Icaria Editorial S. A. 
Weason, M. (2006). Personas en situación de calle: reconocimiento e identidad en contexto de exclusión social. Santiago de Chile: Universidad Alberto Hurtado. Recuperado de http:// repositorio.uahurtado.cl/bitstream/handle/11242/24029/SOCWeason.pdf?sequence=1 